\title{
Mediación de los Objetos Virtuales de Aprendizaje en el Desarrollo de Competencias Matemáticas en Estudiantes de Ingeniería
}

\author{
Olga Martínez-Palmera(1); Harold Combita-Niño(2); Emiro De-La-Hoz-Franco(2) \\ Universidad de la Costa, (1) Departamento de Humanidades, (2) Departamento de Ciencias de la \\ Computación y Electrónica, CUC, Colombia (e-mail: eomartinez@cuc.edu.co; hcombita@cuc.edu.co; \\ edelahoz@cuc.edu.co)
}

Recibido Mar. 1, 2018; Aceptado Abr. 5, 2018; Versión final Jul. 18, 2018, Publicado Dic. 2018

\begin{abstract}
Resumen
El presente artículo analiza la contribución de los Objetos Virtuales de Aprendizaje (OVA) al desarrollo de competencias matemáticas en estudiantes de ingeniería de dos universidades de Barranquilla-Colombia. Se aplicó una prueba diagnóstica a 120 estudiantes representados en dos grupos (control y experimental). Durante un semestre académico se incorporó OVA en el área de cálculo diferencial. Al final del periodo fue aplicada una evaluación que permitió comparar el grado de apropiación de conocimientos matemáticos. Los estudiantes del grupo experimental desarrollaron habilidades matemáticas un $25.9 \%$ por encima del grupo control y el $55 \%$, consideran que son herramientas eficaces para reforzar conocimientos de cálculo diferencial. Se concluyó que la incorporación de OVA al proceso de enseñanza-aprendizaje con la orientación del docente, motiva a los estudiantes por aprender, potencia las habilidades matemáticas de interpretación, modelación de situaciones matemáticas y ejecución de procedimientos para dar solución a distintos problemas de cálculo diferencial.
\end{abstract}

Palabras clave: objeto virtual de aprendizaje; competencias matemáticas; cálculo diferencial; mediación didáctica; ingeniería.

\section{Mediation of Virtual Learning Objects in the Development of Mathematical Competences in Engineering Students}

\begin{abstract}
In the present article the contribution of Virtual Learning Objects (VLO) to the development of mathematical competences in engineering students from two universities in Barranquilla-Colombia is analyzed. A diagnostic test was applied to 120 students represented in two groups (control and experimental). During the academic semester, VLO were incorporated in the differential-calculus subject. At the end of the period a test that allowed to compare the mathematical knowledge was applied. The students of the experimental group developed mathematical skills $25.9 \%$ above the control group, and $55 \%$ consider that Virtual Learning Objects are effective tools to improve differential-calculus knowledge. It was concluded that the incorporation of OVA to teaching-learning process with the teacher's guidance, motivates the students to learn, enhances the mathematical skills of interpretation, mathematical situations modeling and execution of procedures to solve different differential calculus problems.
\end{abstract}

Keywords: learning objects; mathematical competences; differential calculus; didactic mediation; engineering. 


\section{INTRODUCCIÓN}

En el contexto educativo, las Tecnologías de la Información y la Comunicación (TIC) proveen un considerable número de herramientas y escenarios, utilizados en procesos de implementación de estrategias didácticas, con el propósito de facilitar la construcción de conocimiento. Algunos de estos, según (Basantes, Naranjo, Gallegos y Benítez, 2017) son: aulas virtuales, blogs didácticos, evaluaciones online, aprendizaje móvil, realidad virtual y entornos virtuales $3 d$, entre otros. Las TIC han generado un positivo impacto en la educación, mediante la innovación del proceso de enseñanza-aprendizaje. Por ello muchas instituciones educativas se han visto abocadas a incorporarlas en los procesos de formación, en aras de apoyar el fortalecimiento de otras competencias básicas decisivas para el desarrollo humano y los aprendizajes significativos que se apropian en el aula y se aplican en la vida (Arias, 2012). Esto es posible lograrlo, gracias a que la tecnología puede utilizarse para dinamizar la visualización de información, aumentar el acceso explícito a la misma, facilitar su acceso remoto y aumentar el intercambio y la construcción de conocimiento. Es por tanto que desde la aparición y desarrollo de la informática y las telecomunicaciones, todas las actividades humanas han soportado una incesante incorporación tecnológica, siendo la educación una de las actividades con mayor inserción tecnológica en la última década. (Rodríguez et al., 2017). Por tanto, se puede afirmar que integrar las TIC a la docencia puede convertirse en una estrategia adecuada para motivar a los estudiantes (Faúdez et al., 2017), en el presente proyecto más que desarrollar o construir competencias, se pretende generar un ambiente motivacional a través de la utilización herramientas digitales, en el área del cálculo diferencial en los programas académicos de ingeniería.

En el campo de la educación, una de las herramientas digitales más utilizadas son los Objetos Virtuales de Aprendizaje (OVA). Por su adaptabilidad a diversos contextos tecnológicos, por ser recursos propicios para potencializar las competencias de los estudiantes y por su facilidad de uso para complementar el aprendizaje en los cursos presenciales y virtuales. Convirtiéndose en herramientas aliadas dentro y fuera del aula de clases que requieren para su implementación de la formación en el uso pedagógico de las TIC por parte de docentes y estudiantes (Çakiroğlu et al., 2012). Los OVA, hacen parte de esas nuevas tecnologías capaces de innovar en el campo de la educación y han generado que el ser humano inmerso en el proceso de enseñanza aprendizaje se encuentre expuesto a un constante cambio, debido a que la evolución de las tecnologías influencia los procesos de interacción en el campo educativo. (Bernal y Ballesteros, 2017), es por tanto, que la articulación de las nuevas tecnologías con el campo educativo ha de ser un encuentro constructivo, que aporte una herramienta útil y agradable por su papel innovador y motivante.

La educación soportada en la tecnología se ha visto influenciada por el desarrollo de conceptos y el surgimiento de nuevas metodologías permitiendo experimentar cambios significativos como consecuencia de los avances tecnológicos, los cuales también se ven reflejados en la conceptualización de los OVA. Tal es el caso de autores como Wiley (2000), Polsani (2006) y L'Allier (1997), quienes consideran que los OVA son una entidad digital creada para la apropiación de conocimientos, habilidades, actitudes y valores, que cobra sentido en función del sujeto que lo usa. Otros autores como Ossadón y Castillo (2006), consideran que los OVA se constituyen en herramientas fundamentales de apoyo al proceso de enseñanza-aprendizaje, razón por la cual, se hace necesario profundizar en el estudio de estos recursos; todo ello en atención al precepto de que "los problemas de aprendizaje no se limitan sólo a las dificultades que puedan presentar los estudiantes, sino que hay que compartirlos con la forma de enseñanza". Los objetos virtuales de aprendizaje son útiles para lograr una educación más innovadora y pertinente apoyada en el uso pedagógico de las Tecnologías de la Información y las Comunicaciones (TIC). (Ministerio de Educación Nacional, 2010). Es decir, el proceso de aprendizaje se hace más dinámico e interactivo debido a la presentación de contenidos de manera agradable y al desarrollo de actividades de aprendizaje para poner en práctica lo aprendido.

Pese a la importancia los OVA, en la educación, estas herramientas, no todos los OVA incorporados al acto educativo inciden en el aprendizaje de los estudiantes, debido a que no cumplen con los criterios establecidos en los estándares de diseño, obedeciendo muchas veces sólo al deseo de la institución por innovar tecnológicamente. Es decir, la escasa adecuación de estos OVA a las normas de calidad en el diseño tiene una influencia directa en la investigación y la reutilización de los objetos de aprendizaje (Vieira et al., 2016). Por tanto, en pro de crear Objetos Virtuales con contenidos digitales pertinentes y de calidad, se debe formar e incentivar a los docentes en el proceso de aplicación de técnicas de valoración y revisión de los recursos digitales, antes de ser incorporados al proceso educativo. En particular, en las actividades de enseñanza y aprendizaje de las disciplinas afines a la ingeniería, donde se requiere que estos contenidos además de promover el aprendizaje contribuyan al fortalecimiento de competencias interpretativas, argumentativas y propositivas en los estudiantes (Parra et al., 2010). En este sentido se tiene que uno de los principales ejes estratégicos propuestos para el refuerzo académico a estudiantes y desarrollo de los docentes en el nivel universitario es el diseño e implementación de objetos virtuales de aprendizaje (OVA). Para las áreas de matemáticas, química, informática básica, inglés básico, técnicas de estudio, escritura y lectura crítica, esto busca además de contribuir notoriamente en la permanencia de los estudiantes, contribuir al mejoramiento 
de habilidades básicas (Pascuas, Jaramillo y Verástegui. 2015). En este sentido, incorporar un Objeto Virtual de Aprendizaje durante un proceso de formación en Ingeniería, implica considerar esencialmente diferentes estilos de aprendizaje y diferentes saberes conceptuales, procedimentales y propositivos, ya que de allí se derivan distintos propósitos y formas de aprehensión del conocimiento.

En cuanto al aprendizaje en el área de las matemáticas, en Colombia se evidencia que un alto porcentaje de los estudiantes que ingresan a la educación superior presentan dificultades en esta área, específicamente en la interpretación y modelación de problemas, situación que de acuerdo con el criterio de expertos se refleja en los bajos resultados académicos obtenidos en las pruebas de evaluación del aprendizaje y calidad como: Exámenes de Estado de Calidad de la Educación Superior SABER PRO y el Examen de Ciencias Básicas (EXIM),este último es realizado por la Asociación Colombiana de Facultades de Ingeniería (ACOFI). Esta problemática también se ha evidenciado en otros países, como en Estados Unidos, destacándose en diversos informes, el bajo rendimiento en matemáticas. (Mathematical Sciences Education Board, 2004).Por su parte, en el nivel universitario puede caracterizarse la metáfora del estudiante dependiente del profesor, como individuo expectante de que este le explique, le anticipe y le indique "todo" lo que debe hacer, poco predispuesto a enfrentarse a cuestiones y problemas para ser resueltos por él mismo (Corica, 2009). Es por esto que detectar tempranamente a los estudiantes más vulnerables y concentrar la atención en ellos, con una motivación especial a través de diversas estrategias que produzcan buenos resultados para lograr que los estudiantes finalicen con éxito sus estudios. (Pascuas, Jaramillo y Verástegui. 2015). Es una alternativa que se además de ser necesaria requiere de herramientas alternativas y los OVA surgen como una de ellas.

Para mitigar la problemática anteriormente expresada, en el campo educativo, se han llevado a cabo investigaciones tendientes a reorientar los procesos de enseñanza-aprendizaje de las matemáticas, y de esta forma desarrollar una comprensión de las necesidades de estudiantes con dificultades en esta área. Es así como para el desarrollo de las competencias específicas en matemáticas, es esencial vincular los objetivos curriculares con los materiales y actividades empleados por el profesor en el aula (García y Benítez, 2012). Complementariamente se han realizado otras investigaciones, con el fin de promover una mejor actitud por parte de los estudiantes hacia el aprendizaje de las ciencias básicas, mediante el uso de didácticas, que posibiliten el diseño innovador y la utilización de diferentes estrategias y herramientas de apoyo a los estudiantes, en la apropiación de estructuras conceptuales y simbólicas propias de las matemáticas (Gutiérrez et al., 2014). En consecuencia, los OVA pueden asociarse a éste tipo de herramientas, debido a que propician el desarrollo de actividades didácticas en el ámbito de las matemáticas. El uso de la tecnología en la enseñanza de las matemáticas estimula el desarrollo de habilidades para la resolución de problemas y la comprensión de los conceptos matemáticos. Sin embargo, los docentes deben fortalecer sus habilidades en relación al uso de las herramientas tecnológicas.

Es importante destacar que a medida que los profesores exploran y utilizan las herramientas tecnológicas disponibles (relevantes para la enseñanza de las matemáticas), aumenta la motivación por continuar desarrollándose profesionalmente en el uso de la tecnología de apoyo a la enseñanza en el aula (Hartsell et al., 2010).En correspondencia con lo anterior, el bajo rendimiento de los estudiantes en el área de matemáticas puede desencadenaren problemas de baja autoestima, deterioro de la confianza y falta de interés. Tratar de resolver estos problemas sin entender los conceptos subyacentes, crea estudiantes pasivos. Experimentar repetidamente sentimientos negativos, como fracaso, ansiedad o estrés, puede impactar de forma negativa en el desarrollo cognitivo del estudiante. Por tal motivo los juegos educativos se consolidan como herramientas de aprendizaje que benefician el proceso de enseñanza gracias a la motivación generada (Nilsson y Pareto, 2010). Hoy en día las Tecnologías de la Información y la Comunicación TIC en educación, hacen que la informática y los métodos de trabajo, además de integrar la ciencia y la tecnología hayan pasado a formar parte de la vida cotidiana, y por ende afianzar la necesidad de su dominio, razón por la cual estas nuevas y renovadas tecnologías se consideran como un elemento básico en los procesos educativos y afectan a los actores (estudiantes y profesores), quienes no sólo deben tener conocimiento de herramientas informáticas, sino también hacer un uso adecuado de que permitan afianzar el conocimiento adquirido en la parte teórica, y avanzar hacia actividades significativas para el estudiante . (Cabrera, Sánchez, y Rojas. 2016).

Se ha evidenciado que los estudiantes, al interactuar con Objetos Virtuales de Aprendizaje y Software de apoyo al proceso de aprendizaje, cambian su concepción frente a la visión mecánica de la asignatura, ya que al usar herramientas que permiten hacer cálculos y modelar situaciones concretas, posibilita dedicar más tiempo a la generación y afianzamiento del conocimiento, analizando casos concretos en los cuales se socializan los procedimientos, las respuestas y las posibles aplicaciones que estos desarrollos temáticos pueden tener en situaciones propias de sus campos específicos de estudio (Vega et al., 2015).

En el contexto colombiano se evidencia la importancia de implementar didácticas innovadoras para la enseñanza de las matemáticas, que conlleven a la apropiación del conocimiento por parte de los estudiantes, teniendo en cuenta que su aprendizaje se reviste de gran dificultad. Situación que se ve reflejada en los 
resultados obtenidos por los estudiantes en el área de matemáticas, lo cuales demuestran que el sistema educativo colombiano no va al ritmo de los cambios del entorno académico ni de los que imponen las tecnologías de la información y la comunicación. En el año 2012 Colombia participó por tercera vez en el programa Internacional para la evaluación de estudiantes (PISA, por su sigla en inglés), obteniendo un puntaje de 376 puntos, ubicándola muy por debajo de los resultados obtenidos por 61 países. Estos resultados han permanecido estables desde su primera participación en PISA en el año 2006 (ICFES, 2015). En la actualidad y pese a la existencia de investigaciones relacionadas con los OVA, muy pocas están enfocadas en el área de las matemáticas, y en éstas no se analiza la contribución que hacen los OVA al desarrollo de habilidades y competencias básicas, más específicamente muchos de los OVA encontrados no cumplen con estándares reconocidos internacionalmente en relación a su diseño. A esto se le suma que la enseñanza de las matemáticas tiene sus tradiciones, establecidas a lo largo de los siglos y glorificadas por los profesores tradicionales, que en muchos casos van en contravía con las posibilidades ofrecidas por las Tecnologías de la Información y la Comunicación - TIC (Sojka y Pich, 2008). Es necesario anotar que los programas interactivos intentan transformar el aula, agregando un ambiente favorable al proceso de enseñanzaaprendizaje y permitiendo que los educadores sean más receptivos a las nuevas tecnologías en sintonía con las necesidades e intereses de los estudiantes, invitando a una evolución necesaria en el desarrollo de las clases de matemáticas.

Bajo este contexto, se consideró pertinente desarrollar una investigación con el fin de analizar la pertinencia de los OVA en la mediación didáctica, orientados a promover las competencias matemáticas en estudiantes de ingeniería, debido a la evidente relación de las ciencias básicas con la formación integral de los profesionales en ingeniería y a su contribución en el logro de su perfil profesional. Este hecho impulsa a focalizar la mirada hacia la consecución de un aprendizaje significativo por parte del estudiante. Actualmente no basta con el conocimiento literal de los conceptos y fundamentos de los temas tratados, se requiere además que el estudiante sea capaz de aplicar los saberes aprendidos para resolver problemas dentro de un contexto específico. En este orden de ideas, para que las TIC logren impactar en la configuración de nuevos modos de enseñanza y aprendizaje, se requiere de una visión integradora de las políticas educativas, la organización de la institución, los recursos materiales y los actores involucrados que se inscriban en el desarrollo de un proyecto educativo claramente definido y compartido. La complejidad de los desafíos que enfrentan las sociedades globales requerirá soluciones técnicas sofisticadas y una expansión dramática del conocimiento que conduzca al uso de nuevas tecnologías para el abordaje de problemas globales (Wiesner, 2008), por esto en la actualidad se reconoce la importancia de trascender los debates en relación con los aspectos técnicos de las TIC (acceso, cobertura, velocidad) y acercarse a la aplicación de las estrategias educativas que permitan transformaciones en el proceso de enseñanza aprendizaje de los estudiantes que apoyados en las nuevas tecnología avancen hacia nuevas formas de desarrollar el conocimiento. Por lo que los docentes deben apropiarse de las TIC en su práctica educativa. (Valencia, et al 2016) en beneficio de la inclusión de las tecnologías hacia una mejor calidad en la educación. En el 2006 el Ministerio de Educación Nacional (MEN) colombiano, definió el término competencia como "el saber hacer en situaciones concretas que requieren la aplicación creativa, flexible y responsable de conocimientos, habilidades y actitudes" (Ministerio de Educación Nacional, 2006). Complementariamente ha manifestado que "Tener una competencia es usar el conocimiento para aplicarlo a la solución de situaciones nuevas o imprevistas, fuera del aula, en contextos diferentes y para desempeñarse de manera eficiente en la vida personal, intelectual, social, ciudadana y laboral." (Ministerio de Educación Nacional, 2006).

Esto implica que el proceso de enseñanza-aprendizaje por competencias se constituye en una alternativa para el diseño curricular fundamentado en los principios constructivistas y en el aprendizaje significativo; es por esto que la educación debe encaminarse en desarrollar un saber y aplicarlo en situaciones de la vida real y más concretamente resolución de problemas en la práctica. (Marrero, 2017), por lo que se puede afirmar que la escuela tienen dentro de sus funciones, además de contribuir al desarrollo pleno de la persona, formar ciudadanos con posibilidades para poder insertarse en la vida productiva, razón por la que la escuela tiene el compromiso de crear condiciones para que los individuos estén caracterizados y en condición de adaptarse en la actualidad por un entorno global y dinámico, ésta, entre otras características, han sustentado cambios en los planes de estudio en los diferentes niveles de formación a nivel mundial, principalmente en el enfoque centrado en el desarrollo de competencias desde perspectivas constructivistas, con la finalidad de que aquello que se aprende, sirva para poder actuar de forma eficiente ante una situación concreta y determinada. (Trujillo 2014).

Por otra parte, en el módulo de razonamiento cuantitativo de las pruebas Saber Pro, se establece que las competencias relacionadas con las habilidades matemáticas con que los estudiantes universitarios deben contar para desempeñarse adecuadamente en contextos cotidianos independientemente de su carrera son: interpretación y representación, que involucra la comprensión de piezas de información, así como la generación de representaciones diversas a partir de ellas; formulación y ejecución, que involucra procesos relacionados con la identificación del problema, la proposición y construcción de estrategias adecuadas para 
su solución; además de la modelación y el uso de herramientas cuantitativas (aritméticas, métricas, geométricas, algebraicas elementales, y de probabilidad y estadística) y la argumentación que incluye procesos relacionados con la validación de afirmaciones, como lo son justificar o refutar resultados, hipótesis o conclusiones que se derivan de la interpretación y de la modelación de situaciones (ICFES, 2015)

En ese sentido, García (2011), plantea la necesidad de desarrollar algunas habilidades matemáticas incorporando las TIC, documentar y analizar los tipos de razonamiento que emergen en los estudiantes cuando resuelven problemas de matemáticas e interactúan en un ambiente e-learning. Destaca dos tipos de razonamiento en el trabajo de los estudiantes (razonamiento basado en el contexto y en restricciones) los cuales permiten definir las competencias relacionadas con el uso de tecnología que requieren los estudiantes para trabajar en un ambiente virtual de aprendizaje. Los resultados obtenidos a partir de esta investigación se constituyeron en aportes fundamentales para el objeto investigado, debido a que fueron definidas las competencias matemáticas a lograr, previo al diseño de un OVA en la asignatura de Cálculo Diferencial.

Los Objetos de Aprendizaje (LO, por su sigla en inglés) han surgido como un enfoque fundamental para el desarrollo y la distribución de contenido educativo. Estos recursos se almacenan en repositorios de objetos de aprendizaje (LOR), que pueden facilitar a los usuarios la búsqueda de LO mediante el uso de sistemas de recomendación (RS) (Gordillo y Quemada, 2017). Las posibilidades de usar LO en Matemáticas se dan porque la enseñanza de esto implica el reconocimiento de diversos objetos por parte de la resolución de problemas. Eso permite formular nuevos problemas y adaptarlos a cada circunstancia educativa. Las dificultades en el uso de LO se dan en relación a los aspectos culturales y a la misma organización docente, dificultando en el profesor la gestión de las nuevas formas de utilización y redistribución del conocimiento en la era de Internet, porque incluso si existen LO, éstos no son manejados por los maestros (Borja et al., 2017).

En este sentido, la concepción constructivista se organiza en torno a tres ideas fundamentales: (1) el individuo es el responsable último de su propio proceso de aprendizaje, es él quien construye el cocimiento y nadie puede sustituirle en esa tarea, si él no lo hace, nadie, ni siquiera el maestro, puede hacerlo en su lugar; (2)la enseñanza está totalmente mediatizada por la actividad mental constructiva del individuo; y (3) el alumno no es sólo activo cuando manipula, explora, descubre o inventa, además cuando lee o escucha las explicaciones del docente(Briede, 2013). Por otro lado, Ausubel, como otros teóricos cognitivistas, postula que el aprendizaje implica una reestructuración activa de las percepciones, ideas, conceptos y esquemas que el aprendiz posee en su estructura cognitiva. Según (Díaz y Hernández, 2004), se puede caracterizar su postura como constructivista (aprendizaje no es una simple asimilación pasiva de información literal, el sujeto la transforma y estructura) e interaccionista (los materiales de estudio y la información exterior se interrelacionan e interactúan con los esquemas de conocimiento previo y las características personales del aprendiz).

Las necesidades de aprendizaje y las teorías que describen los principios y procesos de aprendizaje, deben reflejar los ambientes sociales subyacentes (Sangpom y Jeerungsuwan, 2014). El conectivismo integra principios de las teorías de caos, redes, complejidad y auto organización. El conectivismo es un referente de gran importancia para esta investigación, debido a que las características que presentan los OVA y la manera en que se da el aprendizaje son afines a los principios de esta teoría. Algunos elementos claves, son: la habilidad del estudiante en ver conexiones, ideas y conceptos, a partir de éstos el estudiante adquiere competencias formando conexiones. El estudiante tiene el control del aprendizaje, decide que aprender, le da significado a la información que recibe. Una de las características de los OVA es la reusabilidad (se pueden usar en varios contextos). La estructura en la que se desarrolla un OVA permite que su contenido pueda cambiarse, acorde con el principio que afirma que la actualización (conocimiento preciso y actual) es la intención de todas las actividades conectivistas de aprendizaje. Otra característica es el nivel de granularidad, que entre más alto sea, permite su utilización en otros OVA de diferentes autores, en este sentido es acorde con los principios del conectivismo.

\section{METODOLOGÍA}

La investigación se fundamentó en el enfoque cuantitativo, de tipo descriptivo-explicativo. Se abordó mediante un diseño cuasi-experimental articulando el componente educativo de habilidades matemáticas, con un componente tecnológico con la mediación de los OVA. Para ello, se conformó un grupo control (60 estudiantes) y un grupo experimental (60 estudiantes), para un total de 120 estudiantes de diferentes programas académicos de ingeniería de Sistemas, Electrónica y Civil pertenecientes a las dos (2) Universidades de Barranquilla-Colombia quienes fueron seleccionados de una población de 400 estudiantes matriculados en Calculo Diferencial de las universidades participantes durante el periodo 2016 (primer semestre). La muestra fue elegida mediante muestreo no probabilístico intencional con la representatividad de cada universidad participante, tomando como referente características homogéneas de estudiantes tales como: estar cursando primer semestre, no ser repitentes, disponibilidad de tiempo y acceso para los estudiantes de ambos grupos control y experimental. 
En el marco de la investigación se definieron tres (3) fases: en la primera fase se aplicó a los estudiantes de ambos grupos control y experimental una prueba diagnóstica en la asignatura cálculo diferencial, antes de iniciar el semestre académico (2016), lo cual permitió identificar el nivel cognitivo y desarrollo de habilidades matemáticas de los estudiantes participantes. En la segunda fase se incorporó al proceso de formación durante el semestre académico dos(2) OVA de cálculo diferencial construidos previamente durante un proyecto denominado Recursos Educativos Digitales Abiertos(REDA) realizado por el Ministerio de Educación Nacional en Colombia denominado Estrategia Nacional REDA durante al año 2015, en conjunto con varias Universidades del país de la cual hicieron parte las dos(2) Universidades del proyecto, fueron aplicados como recurso complementario a las temáticas de la asignatura. Los OVA se estructuraron según se muestra en la figura 3, con base a lo cual fueron aplicados a los estudiantes, y en la tercera fase (al terminar el periodo académico), se aplicó a ambos grupos experimental y de control, una prueba final sobre cálculo diferencial, a fin de contrastar los logros y avances en la apropiación de contenidos de las temáticas y el desarrollo de habilidades matemáticas de los estudiantes y por último, para comparar los resultados de ambos grupos.

Para la recogida de la información, al iniciar el semestre académico (2016), se les aplicó a la muestra una prueba diagnóstica como punto de referencia inicial, diseñada con 20 preguntas distribuidas en cinco unidades de formación: operaciones con fraccionarios, potenciación-radicación, productos notablesfactorización, inecuaciones, límite y derivada. Esta prueba permitió identificar el nivel cognitivo, de apropiación y desarrollo de habilidades matemáticas presentadas por los estudiantes antes de comenzar el desarrollo formal de la asignatura, luego durante su proceso de aprendizaje le fue incorporado el uso de los OVA de Calculo Diferencial como complemento a las temáticas de la asignatura. Al finalizar el semestre, se les aplicó una prueba final que permitió contrastar los logros y avances en la apropiación de contenidos de las temáticas y desarrollo de habilidades matemáticas de los estudiantes.

Cabe destacar que se realizó un consentimiento informado a los estudiantes antes de la aplicación de los instrumentos pretest, donde se les dio a conocer sobre los objetivos del estudio, los beneficios, las alternativas, derechos y responsabilidades de la investigación y estos expresaron voluntariamente su intención de participar en ella después de haber comprendido la información que se le había dado. Para el análisis de los resultados obtenidos se definió una escala de valoración de los conocimientos (bajo, medio, alto y sobresaliente), lo cual permitió la medición de los niveles de conocimientos específicos en el área de matemáticas de los estudiantes. La fiabilidad de la consistencia interna del instrumento pretest se evalúa a través del alfa de Cronbach, obteniéndose un valor de 0.81 , que de acuerdo al criterio de fiabilidad de George y Mallery (2003, p. 231), se considera que el instrumento elaborado y utilizado para este proyecto es bueno y por ende confiable.

De igual manera, durante el desarrollo del proyecto de investigación, se aplicó a los estudiantes del grupo experimental una ficha de observación no participante con la finalidad de medir el grado de aceptación de los OVA y evaluar aspectos como: conocimiento del OVA, la facilidad de acceso, tiempo de navegabilidad, la motivación del estudiante en el uso del OVA, interactividad con el OVA, entre otros aspectos.

Se utilizó además una Rúbrica de evaluación institucional con el fin de validar las características técnicas y funcionales del OVA, bajo tres categorías de evaluación: Temática, Educativa y categoría Tecnológica con 11 indicadores generales. Este instrumento fue diligenciado por 10 docentes que imparten la asignatura Calculo Diferencial en la metodología presencial de la Facultad de Ingeniería de las Universidades participantes, después de haber navegado y analizado el OVA; con la finalidad de evaluar las características técnicas y funcionales de los OVA. Estas tres categorías están constituidas por 11 indicadores generales, definidos por el Ministerio de Educación Nacional (2012), durante la implementación de la estrategia REDA (Recursos Educativos Digitales Abiertos en las Instituciones de Educación Superior).

\section{RESULTADOS}

Los resultados obtenidos producto de la aplicación de la prueba diagnóstica a los 120 estudiantes de los programas académicos de ingeniería en las dos (2) universidades participantes, fueron analizados mediante una escala de valoración de conocimientos básicos en cálculo diferencial descritos en las tablas 1 y $1 \mathrm{~A}$.

Adicionalmente, en la tabla 2 y figura 1, se presentan un comparativo de los resultados obtenidos producto de la aplicación de las pruebas a los estudiantes de los grupos control y experimental. Con relación a las habilidades matemáticas desarrolladas por los 120 estudiantes de los programas académicos de ingeniería de las universidades participantes evaluadas, se observa un aumento significativo en el porcentaje de apropiación del conocimiento en las temáticas y de las habilidades matemáticas, frente a los resultados obtenidos por los estudiantes del grupo control, en la prueba final. Es decir, según la tabla 2, las habilidades específicas para el grupo experimental "reconoce los diferentes tipos de funciones", pasó de $30.3 \%$ en la 
prueba diagnóstica (pretest) a 58.9\% en la prueba final (postest), que representa un significativo aumento de 28,6 puntos porcentuales. En la competencia "comprende el concepto de derivada", se observa un porcentaje $47.7 \%$ en el pretest frente a un $68,3 \%$ en el postest, lo cual representa un aumento de 20,6 puntos porcentuales. En la tercera competencia "reconoce la importancia de las funciones trascendentes", se evidencia un incremento de 23,9 puntos porcentuales, pasado de $43.9 \%$ a $67.8 \%$ y en cuanto a la competencia "aplica las derivadas en la solución de problemas", se generó un significativo aumento de 30,5 puntos porcentuales, pasando de $28.9 \%$ en la prueba diagnóstica frente a $59.4 \%$ en la prueba final, evidenciándose notables diferencias entre el grupo experimental y el grupo control, dado que en grupo experimental se observa un incremento significativo de los porcentajes en todos los indicadores con relación al grupo control.

Tabla 1: Conocimientos básicos en cálculo diferencial de los estudiantes del grupo control

\begin{tabular}{|c|c|c|c|}
\hline Niveles & Preguntas & $\begin{array}{c}\text { Cantidad de estudiantes } \\
\text { que acertaron }\end{array}$ & $\begin{array}{l}\text { \% de estudiantes } \\
\text { que acertaron }\end{array}$ \\
\hline Bajo & $1-7$ & 24 & 40 \\
\hline Medio & $8-13$ & 32 & 53.33 \\
\hline Alto & $14-17$ & 4 & 6.67 \\
\hline Sobresaliente & $18-20$ & 0 & 0.0 \\
\hline & Totales & 60 & 100.0 \\
\hline
\end{tabular}

Tabla 1 A: Conocimientos básicos en cálculo diferencial de los estudiantes del grupo experimental

\begin{tabular}{|c|c|c|c|}
\hline Niveles & Preguntas & $\begin{array}{c}\text { Cantidad de estudiantes } \\
\text { que acertaron }\end{array}$ & $\begin{array}{c}\text { \% de estudiantes } \\
\text { que acertaron }\end{array}$ \\
\hline Bajo & $1-7$ & 22 & 36.67 \\
\hline Medio & $8-13$ & 35 & 58.33 \\
\hline Alto & $14-17$ & 3 & 5.0 \\
\hline Sobresaliente & $18-20$ & 0 & 0.0 \\
\hline \multicolumn{2}{r}{ Totales } & 60 & 100.0 \\
\hline
\end{tabular}

Tabla 2: Comparativo de las habilidades matemáticas de los estudiantes en la prueba diagnóstica y final

\begin{tabular}{|l|c|c|c|c|c|}
\hline \multirow{2}{*}{ Indicador } & \multicolumn{2}{|c|}{ Test diagnostico } & \multicolumn{2}{c|}{ Test final } & comparativo \\
\cline { 2 - 6 } & $\begin{array}{c}\text { grupo } \\
\text { experimental } \\
\text { /aprobaron }\end{array}$ & $\begin{array}{c}\text { grupo control } \\
\text { / aprobaron }\end{array}$ & $\begin{array}{c}\text { grupo } \\
\text { experimental } \\
\text { /aprobaron }\end{array}$ & $\begin{array}{c}\text { grupo } \\
\text { control } \\
\text { aprobaron }\end{array}$ & $\begin{array}{c}\text { grupo } \\
\text { experimental } \\
\text { vs } \\
\text { grupo control }\end{array}$ \\
\hline $\begin{array}{l}\text { Reconoce los diferentes } \\
\text { tipos de funciones }\end{array}$ & 30.3 & 32.6 & 58.9 & 41.1 & $58.9>41.1$ \\
\hline $\begin{array}{l}\text { Comprende el concepto } \\
\text { de derivada }\end{array}$ & 47.7 & 43.5 & 68.3 & 31.7 & $68.3>31.7$ \\
\hline $\begin{array}{l}\text { Reconoce la importancia } \\
\text { de Ias funciones } \\
\text { trascendentales }\end{array}$ & 43.9 & 40.2 & 67.8 & 32.2 & $67.8>32.2$ \\
\hline $\begin{array}{l}\text { Aplica las derivadas en la } \\
\text { solución de problemas }\end{array}$ & 28.9 & 27.1 & 59.4 & 40.6 & $59.4>40.6$ \\
\hline
\end{tabular}

La aplicación de la rúbrica de evaluación de los OVA por parte de los docentes, permitió identificar el cumplimento de indicadores y las características técnicas y funcionales de cada uno de los OVA analizados en una calificación en el rango de 0-100.En la categoría temática se evidenció que las principales fortalezas estuvieron centradas en el manejo riguroso de la temática $(60 \%)$ y la estructura de los contenidos $(22 \%)$. Sin embargo, se detectaron algunos aspectos por mejorar relacionados con la existencia de aportes del autor $(2 \%)$ y la actualidad de los contenidos (16\%). En la categoría educativa los docentes señalan que los OVA fueron pertinentes a la población a la cual estaba dirigido (36.7\%) y se evidencia coherencia con los objetivos planteados $(50 \%)$. Sin embargo, consideran deficiente que los OVA sintetizan lo que se deseaba transmitir $(13.3 \%)$.

En la categoría tecnológica los docentes estuvieron de acuerdo en que los OVA presentaban una interfaz amigable e intuitiva (51.4\%), el grado de integración de sus componentes (actividades, ejercicios, entre otros) fueron pertinentes a las competencias definidas y a los temas tratados (31.4\%). Consideran los docentes que se debe mejorar la información para facilitar su instalación (2.9\%) y el Nivel de interacción con el usuario (14.3\%). A continuación, en la figura 2, se muestran los resultados obtenidos de la aplicación de la rúbrica de valoración de los OVA. 


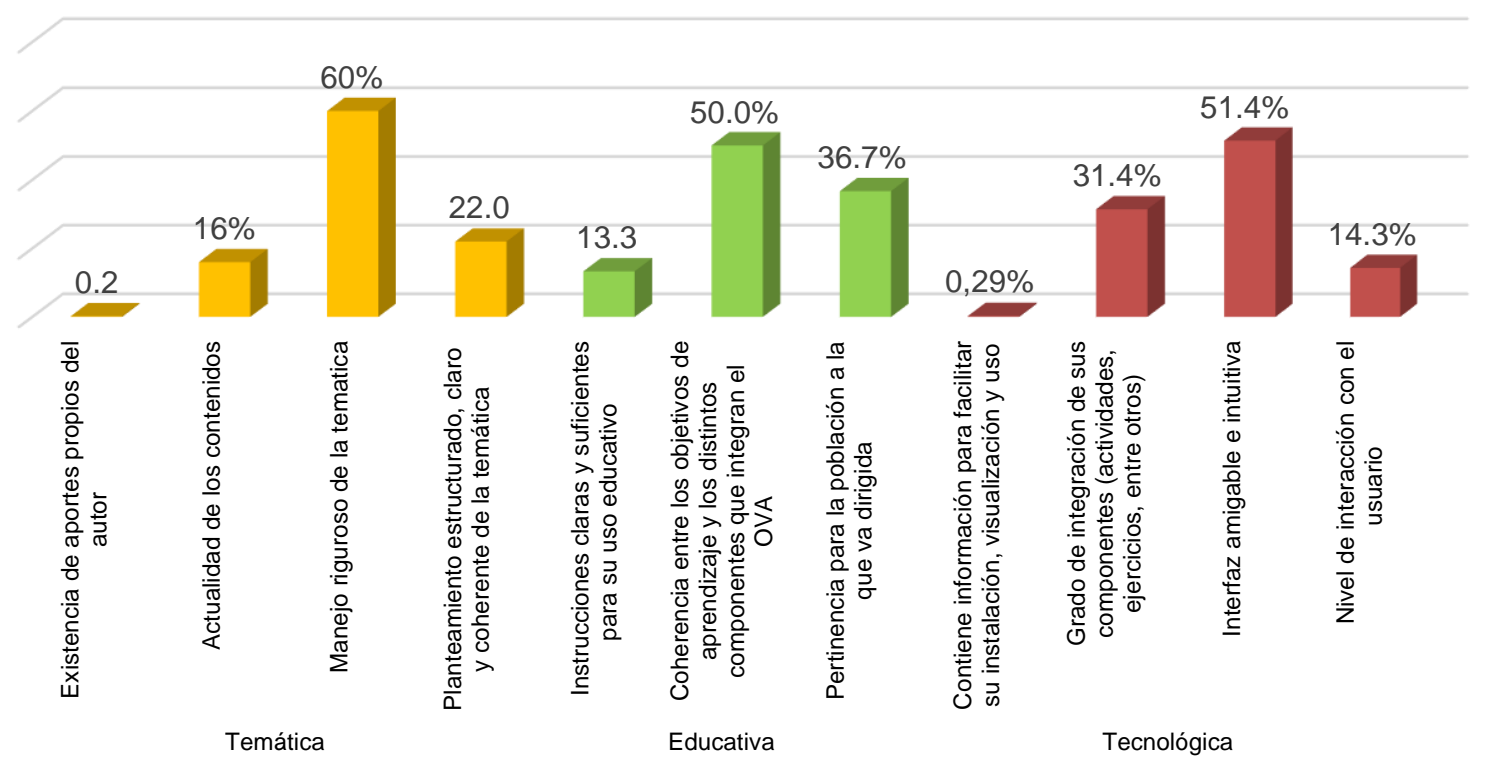

Fig. 1: Resultados de la evaluación de los OVA mediante la aplicación de la rúbrica

De igual manera, con el fin de observar y registrar el comportamiento general de los 120 estudiantes seleccionados, con relación al: nivel de apropiación conceptual, la facilidad de uso, el nivel de interactividad y la aplicabilidad de los conceptos durante el desarrollo de actividades de aprendizaje frente al uso del OVA de cálculo diferencial; se realizó una observación directa-estructurada no participante, utilizando como instrumento una ficha de observación estandarizada que contenía los siguientes aspectos a evaluar: conocimiento del OVA, facilidad de acceso, tiempo de navegabilidad, motivación del estudiante en el uso del OVA e interactividad con el OVA. Los resultados se describen en la tabla 3.

El análisis de los resultados descritos en la tabla 3, señala que el $60 \%$ de los estudiantes participantes desconocían la existencia de los OVA de cálculo diferencial, pese a estar referenciado en la página web Institucional de cada Universidad. El 40\% restante manifestaron conocer la existencia del OVA de cálculo diferencial pero que no habían ingresado a este. Producto de la observación directa, se pudo evidenciar que los OVA son de fácil navegación y rápido acceso por parte de los estudiantes. Sin embargo, los estudiantes han manifestado que debe disminuirse el número de clics que se requieren para acceder a algunos de los OVA analizados, evitando el ingreso a éstos, de forma lineal o secuencial, por ello se sugiere la creación de árboles de navegación. Otro aspecto evaluado fue el tiempo de navegación empleado por cada estudiante para recorrer el OVA, en promedio 45 minutos. De igual manera, el 55\% de los estudiantes se mostraron motivados con el uso de los OVA, afirmaron que es una buena herramienta para reforzar los conocimientos de cálculo diferencial, porque les permite acceder a los contenidos las veces que sea necesario para afianzar y complementar sus conocimientos. Manifiestan además que los OVA son muy didácticos y sencillos, por tanto, crean un ambiente de estudio agradable, con facilidad de uso. Coinciden en que no se requiere de conocimientos avanzados y, por último, consideran que las actividades son coherentes con las temáticas abordadas desde el cálculo diferencial.

Como aspecto a mejorar, los estudiantes manifestaron que los OVA presentan varios errores en las actividades evaluativas, sobre todo en las preguntas de completitud, que no les permitían llenar en su totalidad los espacios y en otros casos quedan faltando espacios para escribir. Sugieren que deben ser más concretas y en algunos casos, el sistema no deja escribir nada en el espacio para completar.

Tabla 3: Recopilación de resultados a partir de la ficha de observación a los estudiantes de Cálculo Diferencial

\begin{tabular}{|l|l|}
\hline \multicolumn{1}{|c|}{ Aspectos evaluados } & \multicolumn{1}{c|}{ Observaciones } \\
\hline $\begin{array}{l}\text { Conocimiento del OVA por } \\
\text { parte de los estudiantes }\end{array}$ & $\begin{array}{l}\text { El 60\% de los estudiantes participantes desconocían la existencia de los OVA de cálculo } \\
\text { diferencial, pese a que estos se encontraban referenciados en la página web Institucional. } \\
\text { El 40\% de los estudiantes que manifestaron conocer la existencia de los OVA por ser de } \\
\text { primer semestre aún no habían accedido a estos. }\end{array}$ \\
\hline $\begin{array}{l}\text { Facilidad de Acceso al } \\
\text { OVA por parte de los } \\
\text { estudiantes }\end{array}$ & $\begin{array}{l}\text { Se evidenció durante la aplicación de los OVA que los estudiantes desarrollaron el proceso } \\
\text { apropiando cada uno de los pasos, con lo que se pudo concluir que el acceso a los OVA } \\
\text { utilizado es amigable y fácil de navegar. Este hecho generó motivación en los estudiantes } \\
\text { por profundizar los temas propuestos. }\end{array}$ \\
\hline
\end{tabular}


Tabla 3 (continuación)

\begin{tabular}{|l|l|}
\hline $\begin{array}{l}\text { Tiempo de navegabilidad } \\
\text { del estudiante en el OVA }\end{array}$ & $\begin{array}{l}\text { En promedio, el tiempo para la navegabilidad requerido por los estudiantes, para revisar } \\
\text { todo el contenido de los OVA fue de 45 minutos, un tiempo menor al previsto con lo que } \\
\text { se pudo observar que la herramienta incremento el interés y consecuentemente permitió } \\
\text { la revisión de una cantidad mayor de información y el desarrollo de actividades de } \\
\text { aprendizaje contenidas en el OVA. }\end{array}$ \\
\hline $\begin{array}{l}\text { Motivación del estudiante } \\
\text { en el uso del OVA }\end{array}$ & $\begin{array}{l}\text { Según la ficha de observación, se dedujo que el 55\% de los estudiantes se mostraron } \\
\text { motivados con el uso de los OVA, además se observó que en este porcentaje los } \\
\text { estudiantes afirmaron que es una buena herramienta para reforzar y complementar los } \\
\text { conocimientos adquiridos en relación al Cálculo Diferencial. Los OVA son útiles para } \\
\text { aclarar dudas y subsanar falencias, dado que son muy didácticos y sencillos de utilizar, } \\
\text { generando un ambiente de estudio agradable. }\end{array}$ \\
\hline Interactividad con el OVA & $\begin{array}{l}\text { Durante la interactividad de los estudiantes con los OVA, se evidenció que los estudiantes } \\
\text { aplicaban varias veces la misma prueba para obtener el resultado correcto de las } \\
\text { actividades de aprendizaje y validar su respuesta, pero a su vez sugirieron que las } \\
\text { respuestas a las validaciones se deberán mostrar en una página emergente y permitirles } \\
\text { regresar a cualquier etapa del proceso. }\end{array}$ \\
\hline $\begin{array}{l}\text { Destreza del estudiante } \\
\text { para acceder al OVA }\end{array}$ & $\begin{array}{l}\text { Se observó que los estudiantes contaban con destrezas para acceder a los OVA } \\
\text { propuestos, evidenciándose que con unos conocimientos básicos abordaban los } \\
\text { contenidos a través de estos recursos educativos digitales. }\end{array}$ \\
\hline $\begin{array}{l}\text { Coherencia de las } \\
\text { actividades con los } \\
\text { contenidos }\end{array}$ & $\begin{array}{l}\text { Los estudiantes sugieren que las actividades evaluativas deberían ser más concretas y en } \\
\text { algunos casos, el sistema no deja escribir nada en el espacio de complete. Consideran } \\
\text { que las actividades son coherentes con las temáticas abordadas desde el cálculo } \\
\text { diferencial. Los OVA solo contienen dos tipos de actividades evaluativas de arrastrar y } \\
\text { completar. }\end{array}$ \\
\hline
\end{tabular}

La identificación de las habilidades específicas en la asignatura de cálculo diferencial en las universidades participantes, se describen en la tabla 4.

Tabla 4: Habilidades específicas y unidades de formación de la asignatura de Cálculo Diferencial

\begin{tabular}{|l|l|}
\hline \multicolumn{1}{|c|}{ Habilidades específicas en cálculo diferencial } & Unidades de formación \\
\hline $\begin{array}{l}\text { Reconocer los diferentes tipos de funciones, aplicarlos en la } \\
\text { construcción de modelos matemáticos de fenómenos del mundo } \\
\text { real; particularmente en las ciencias básicas de la ingeniería y } \\
\text { comprender el concepto de límite de una función y su importancia } \\
\text { en la construcción del Cálculo Diferencial. }\end{array}$ & Funciones, límites y continuidad \\
\hline $\begin{array}{l}\text { Comprender el concepto de la derivada de funciones y desarrollar } \\
\text { habilidades y destrezas en la aplicación de las reglas de } \\
\text { derivación utilizadas en problemas de aplicación relacionados con } \\
\text { la física, la ingeniería y la química. }\end{array}$ & Concepto de derivada y reglas de derivación \\
\hline $\begin{array}{l}\text { Reconocer la importancia de las funciones trascendentes en el } \\
\text { modelado de fenómenos del mundo real y aplicar el Cálculo } \\
\text { Diferencial en la solución de problemas en las ciencias básicas de } \\
\text { la ingeniería. }\end{array}$ & Aplicaciones de la derivada (ingeniería) \\
\hline $\begin{array}{l}\text { Aplicar las derivadas en la solución de problemas sobre rectas } \\
\text { tangentes y normales, razones de cambio relacionadas y } \\
\text { problemas de optimización. }\end{array}$ & Aplicaciones de la derivada \\
\hline $\begin{array}{l}\text { Construir la gráfica de una función a partir de los criterios de la 1a } \\
\text { y } 2^{a} \text { derivada. }\end{array}$ & Aplicaciones de la derivada \\
\hline
\end{tabular}

La tabla 4 contiene las competencias específicas y unidades de formación comunes que fueron incluidas en los planes de asignatura de cálculo diferencial por las universidades participantes y otras universidades analizadas en el orden nacional. Estas competencias son evaluadas en las pruebas EXIM de ACOFI y Saber Pro del MEN. Por tanto, los OVA aplicados estuvieron orientados a desarrollar acciones encaminadas a fortalecer estas competencias, lográndose el objetivo planteado en la investigación. Los OVA utilizados en este trabajo de investigación, fueron desarrollados por un grupo de docentes constructores de contenidos del área de ciencias básicas, más específicamente de la asignatura de cálculo diferencial. Ellos han tomado como referentes las competencias definidas en la asignatura de cálculo diferencial de diversas universidades del país, agrupadas bajo cinco (5) categorías, consideradas como las de mayor dificultad de aprendizaje, para lo 
cual se procedió a crear los contenidos de refuerzo que fueron puestos a disposición de los estudiantes. En la figura 2, se muestra la página de ingreso a uno de los OVA desarrollados en la asignatura de cálculo diferencial, durante el trabajo de investigación.

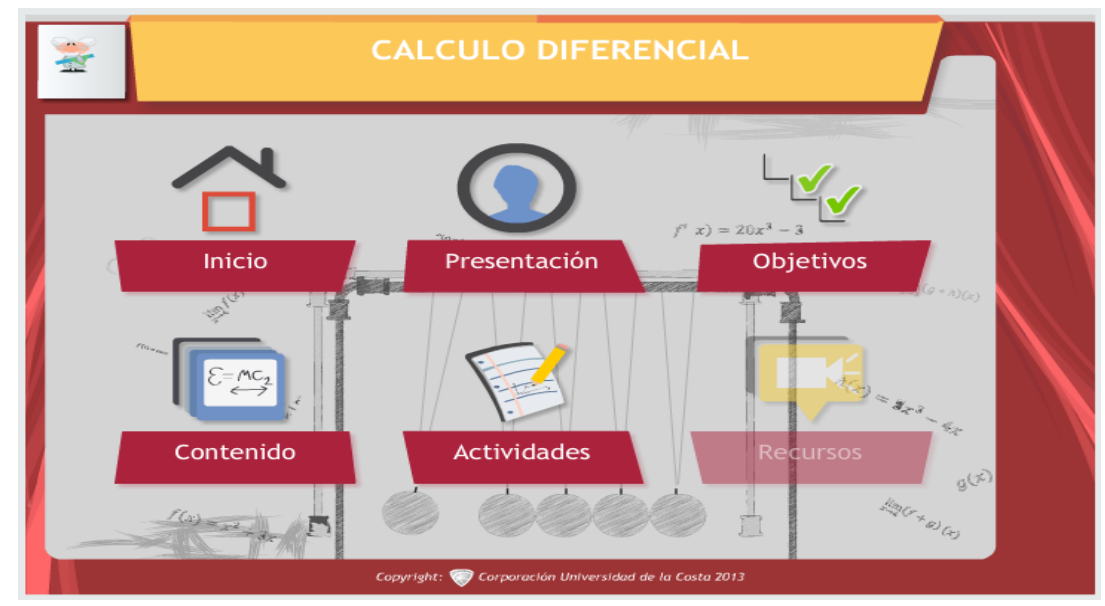

Fig.2: Página de acceso a un OVA de Calculo Diferencial

Al acceder a cada una de las pestañas se encuentra la siguiente información:

Inicio (título del OVA, descripción del OVA, indicadores de desempeño, indicaciones de navegación). Objetivos (objetivo de la temática). Contenidos (temas del OVA navegados de manera interactiva bajo formatos multimedia (hipertexto, audio, videos), sub temas (hipertexto, audio, videos) con navegación con navegación tanto lineal como por panel de navegación. Actividades de aprendizaje y evaluativas (actividades de apropiación de la temática, actividades evaluativas interactivas, retroalimentación inmediata, opciones para volver a desarrollar la actividad). En las figuras 3 y 4, se muestra ventanas de acceso a los OVA.

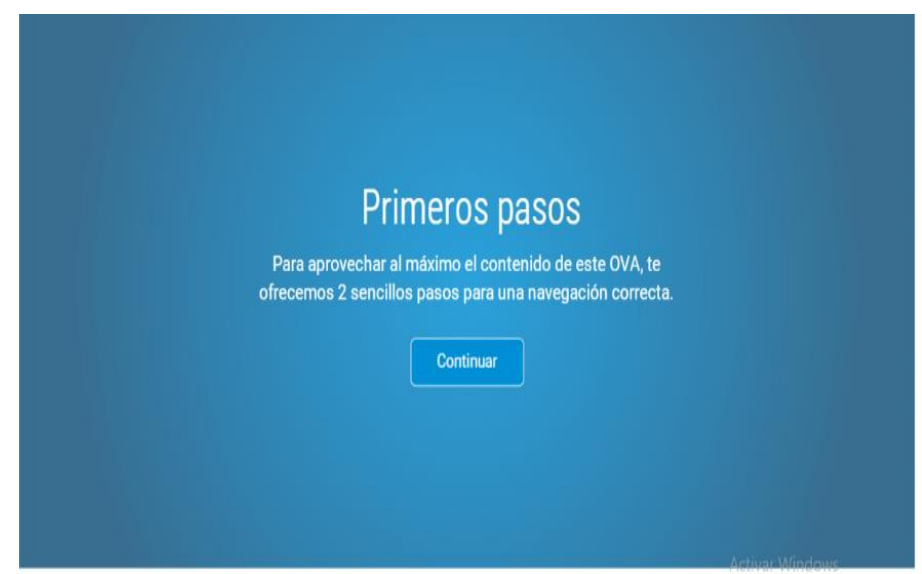

(ANT) Sac)

Fig.3: Página de inicio de acceso a los contenidos del OVA de Calculo Diferencial

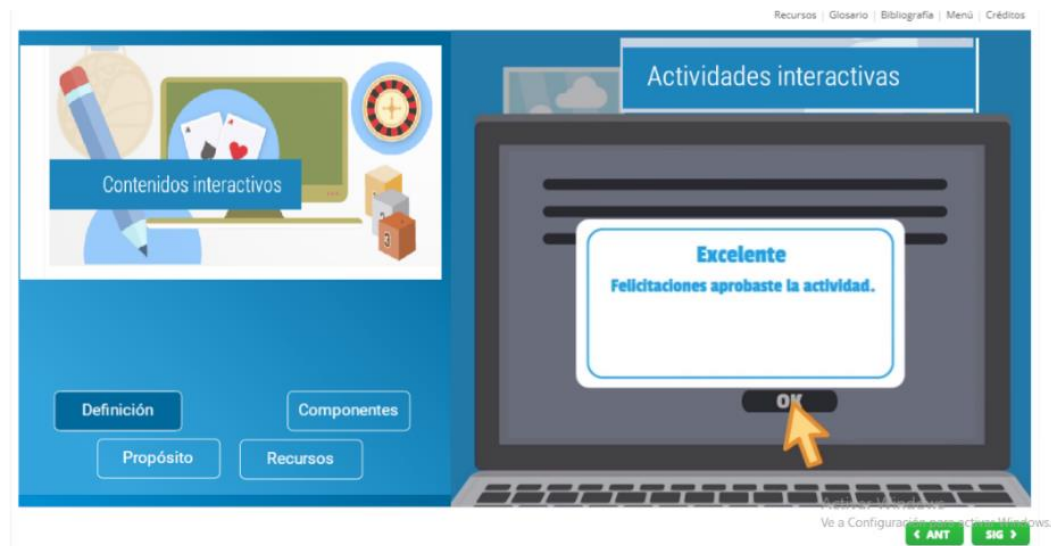

Fig.4: Página de acceso a los contenidos del OVA de Calculo Diferencial 


\section{CONCLUSIONES}

De acuerdo a lo resultados obtenidos en la investigación, se concluye que la incorporación de los OVA en el proceso de enseñanza - aprendizaje con la orientación permanente de los docentes, motiva a los estudiantes por aprender favoreciendo la apropiación del conocimiento al potenciar las habilidades matemáticas de interpretación, modelación de situaciones matemáticas y ejecución de procedimientos o estrategias para dar solución a distintos problemas en el área de cálculo diferencial, lo cual fortalece el desarrollo de competencias genéricas tanto para su vida profesional como personal. De igual manera la inclusión de OVA como medicación didáctica mejora la práctica pedagógica de los docentes de ingeniería durante la enseñanza del cálculo diferencial.

A los docentes se les facilitó la aplicación de los OVA de cálculo diferencial, debido a que estos participaron de manera preliminar en el proceso de construcción de los mismos, en adición y debido a la aplicación del piloto con los estudiantes y la rúbrica de evaluación les permitió proponer mejoras a los OVA con base en los resultados obtenidos de los estudiantes. Los OVA utilizados durante el proceso investigativo contenían retroalimentación durante el desarrollo de actividades de aprendizaje por parte de los estudiantes, sin embargo las sugerencias de los estudiantes fueron tenidas en cuenta para enriquecerlos a futuras aplicaciones. Debido a que el proceso fue exitoso, se recomienda su implementación tanto en las Universidades participante como en otras instituciones que lo requieran.

Por último, es importante resaltar que los aspectos de mayor motivación para propiciar la usabilidad de los OVA por parte de los estudiantes son la calidad de diseño, facilidad de acceso, presentación estructurada de los contenidos con acceso no lineal y la existencia de mecanismos de retroalimentación del aprendizaje.

Cabe resaltar que los aspectos de mayor motivación para propiciar la usabilidad de los OVA por parte de los estudiantes son la calidad de diseño, facilidad de acceso, presentación estructurada de los contenidos con acceso no lineal y la existencia de mecanismos de retroalimentación del aprendizaje.

\section{REFERENCIAS}

Arias, L., Impacto de un curso mediado por la Web 2.0 en el desarrollo profesional de un grupo de futuros docentes de inglés, Folios, 51(36), 51-76 (2012)

Basantes, V., M. Naranjo, M. Gallegos y N. Benítez, Los Dispositivos Móviles en el Proceso de Aprendizaje de la Facultad de Educación Ciencia y Tecnología de la Universidad Técnica del Norte de Ecuador, Formación Universitaria, 10(2), 79-88 (2017)

Bernal, L. y J. Ballesteros, Metodología para la construcción de Objetos Virtuales de Aprendizaje, apoyada en Realidad Aumentada, Sophia, 13(1), 4-12 (2017)

Borja, L., O. Santos y G. Espinoza, Learning Objects in Teaching Mathematics.Revista Publicando, 4(10), $550-558$ (2017)

Briede, B., A constructivist approach in engineering education, Paper presented at the Engineering for Rural Development, 584-589 (2013)

Cabrera, J., I. Sánchez y F. Rojas, Uso de Objetos Virtuales de Aprendizaje OVAS Como estrategia de enseñanzaaprendizaje inclusivo y complementario a los cursos teóricos-prácticos, Educación en Ingeniería, 11(22), 4-12 (2016)

Çakiroğlu, U., A. Baki e Y. Akkan, The effects of using learning objects in two different settings, Turkish Online Journal of Educational Technology, 11(1), 181-19(2013)

Corica, A.R., Aprender Matemática en la Universidad: la perspectiva de estudiantes de primera año, Revista electrónica de investigación en educación en ciencias, 4(1), 10-2 (2009)

Díaz, F. y G. Hernández, Estrategias Docentes para un aprendizaje significativo, M. G. Hill, Ed. (2004)

Faúndez, C., A. Bravo, P. Ramírez y H. Astudillo, Tecnologías de la Información y la Comunicación (TIC) en el Proceso de Enseñanza-Aprendizaje de Conceptos de Termodinámica como Herramienta para Futuros Docentes, Formación Universitaria, 10(4), 43-54 (2017)

García, B., Proyecto Competencias Matemáticas Desarrolladas en Ambientes de Aprendizaje, Formación Universitaria, 4(3) (2011)

García, M., Diseño e Implementación de Tareas para Apoyar el Aprendizaje de las Matematicas, doi:10.4067/S071850062013000100003, Formación Universitaria, 6(1), 13-20(2013)

George, D. y P. Mallery, SPSS for Windows step by step: A Simple Guide and Reference, 11.0 Update, 4ª Ed., Boston, Allyn y Bacon (2003)

Gordillo, A., E. Barra y J. Quemada, A Hybrid Recommendation Model for Learning Object Repositories, IEEE Latin America Transactions, 15(3), 462-473 (2017)

Gutiérrez, L., L. Ariza y J. Jaramillo, Estrategias didácticas en el uso y aplicación de herramientas virtuales para el mejoramiento en la enseñanza del cálculo integral, Revista Academia y Virtualidad, 7 (2), 64 -75 (2014) 
Hartsell, T., S. Herron, H. Fang y A. Rathod, Improving teachers' self-confdence in learning technology skills and math education through professional development, International Journal of Information and Communication Technology Education, 6(2), 47-61 (2010)

ICFES, Módulo de Razonamiento cuantitativo Saber Pro 2015-2 (2015)

L'Allier, J., Frame of Reference: NETg's Map to the Products, Their Structure and Core Beliefs (1997)

Leidner, D. y S. Arvenpaa, The use of information technology to enhance management school education: A theoretical view, MIS Quarterly, Management Information Systems, 19(3), 265-291(1995)

Mathematical Sciences Education Board. On evaluating curricular effectiveness: Judging the quality of K-12 mathematics evaluations, MSEB Publications (2004)

Marrero, O., El proceso de enseñanza-aprendizaje por competencias. Una visión desde el enfoque sistémico. En: Congreso Universidad (2017)

Ministerio de Educación Nacional, ¿Qué es un Objeto Virtual de Aprendizaje? (2010)

Nilsson, A. y L. Pareto, The complexity of integrating technology enhanced learning in special math education - A case study (2010)

Ossandón, Y. y P. Castillo, Propuesta para el diseño de objetos de aprendizaje. Revista Facultad de IngenieríaUniversidad de Tarapacá, 14(1), 36-48(2006)

Parra, E. y A. Narváez, Construcción de objetos virtuales de aprendizaje para ingeniería desde un enfoque basado en problemas, Revista Virtual Universidad Católica del Norte, Septiembre-Diciembre, 84-104 (2010)

Pascuas, Y., C. Jaramillo y F. Verástegui, Desarrollo de objetos virtuales de aprendizaje como estrategia para fomentar la permanencia estudiantil en la educación superior, Revista Escuela de Administración de Negocios, (79) (2015)

Piccoli, G., R. Ahmad y B. Ives, Web-based virtual learning environments: A research framework and a preliminary assessment of effectiveness in basic it skills training, MIS Quarterly: Management Information Systems, 25(4), 401-426 (2001)

Polsani, R., Use and Abuse of Reusable Learning Objects, Journal of Digital Information, (SI.) 3 (4) (2006)

Rivera, D. P., Mathematics education and students with learning disabilities: Introduction to the special series, Journal of Learning Disabilities, 30(1), 2-19 (1997)

Sangpom, N. y N. Jeerungsuwan, The development of an instructional model using connectivism to promote creativity based on teacher professional standard for thai higher education, International Journal of Learning in Higher Education, 20(3), 71-77 (2014)

Sojka, P. y R. Plch, Technological challenges of teaching mathematics in a blended learning environment, International Journal of Continuing Engineering Education and Life Long Learning, 18(5-6), 657-665(2008)

Trujillo, J., El enfoque en competencias y la mejora de la educación, Ra Ximhai, 10(5) (2014)

Valencia, T., A. Serna y otros cuatro autores, Competencias y estándares TIC desde la dimensión pedagógica: una perspectiva desde los niveles de apropiación de las TIC en la práctica educativa docente (2016)

Vega, J., F. Niño e Y. Cárdenas, Enseñanza de las matemáticas básicas en un entorno e-Learning: un estudio de caso de la Universidad Manuela Beltrán Virtual, Revista EAN, (79), 172-187 (2015)

Vieira, M., M. Morales y J. Rossato, Evaluation of Virtual Objects: Contributions for the Learning Process. The International Review of Research in Open and Distributed Learning, 17(6) (2016)

Wiesner, T. y W. Lan, Technology in engineering education-essential for sustainable approaches to global technical challenges, World Review of Science, Technology and Sustainable Development, 5(3-4), 212-233(2008)

Wiley, D., Connecting learning objects to instructional design theory: a definition, a metaphor, and a taxonomy, Utah State University, Digital Learning Environments Research Group, (435) 797-7562 (2000) 